\title{
PERFIL DOS PACIENTES ATENDIDOS NO SETOR DE FONOAUDIOLOGIA DE UM SERVIÇO PÚBLICO DE RECIFE - PE
}

\author{
Profile of patients receiving speech therapy care \\ at a public service at Recife-PE
}

Percy Maria de Lima Barros ${ }^{(1)}$, Priscila Nogueira de Oliveira ${ }^{(2)}$

\begin{abstract}
RESUMO
Objetivo: traçar o perfil dos pacientes cadastrados e atendidos pelo setor de Fonoaudiologia de um serviço público de Recife. Métodos: o estudo foi realizado a partir da coleta e análise dos dados referentes à origem do encaminhamento, faixa etária, gênero, queixas fonoaudiológicas de crianças, adolescentes, adultos e idosos, colhidos das fichas das entrevistas iniciais. A amostra foi constituída por todos os indivíduos cadastrados no setor de Fonoaudiologia de um serviço público da cidade de Recife, no período de julho de 2007 a maio de 2008. Resultados: dos 251 pacientes atendidos, 12 foram excluídos da pesquisa, três destes obtiveram alta fonoaudiológica e nove não apresentavam os dados completos. Das fichas analisadas, houve um maior número de encaminhamento realizado por Pediatras (25\%), em seguida Otorrinolaringologista (16\%) e Clínico Geral (10\%), sendo a maioria do sexo masculino (61\%), com idade entre 0 a 11 anos de idade. De acordo com as queixas, as mais freqüentes foram de desvio fonológico (14\%), seguidos de atraso de linguagem (11\%), disfluência $(10 \%)$ e respirador oral (8\%). Em relação ao número de queixas apresentadas, $72 \%$ apresentaram uma queixa, $25 \%$ duas queixas e $2 \%$ três queixas associadas. Conclusões: o perfil atendido no setor de fonoaudiologia de um serviço público da cidade de Recife, apesar de ser variado, é em sua maioria do sexo masculino, encaminhado por pediatras, com alteração de voz e idade entre 0 a 11 anos, apresentando frequentemente uma única queixa fonoaudiológica.
\end{abstract}

DESCRITORES: Atenção Primária à Saúde; Saúde Pública; Setor Público; Serviços de Saúde; Reabilitação

\section{INTRODUÇÃO}

O Sistema Único de Saúde (SUS) tem como um de seus princípios básicos a universalidade no atendimento de saúde, garantindo assim, acesso aos serviços através das entidades conveniadas ao sistema a todos os usuários, integrando-se aos princípios da eqüidade, integralidade, resolutividade e gratuidade, buscando soluções para melhoria da qualidade de vida da população ${ }^{1}$.

A inserção do fonoaudiólogo na saúde coletiva requer um profissional comprometido com os

(1) Fonoaudióloga do Instituto de Reabilitação Infantil, Recife, PE; Pós-Graduanda em Fonoaudiologia na Saúde Pública pela Universidade de Pernambuco.

(2) Fonoaudióloga residente da Residência Multiprofissional em Saúde do Instituto de Medicina Integral Professor Fernando Figueira, IMIP, Recife, PE.

Conflito de interesses: inexistente princípios de humanização, acolhimento, vínculo e responsabilidade pelo território em que atua e pela comunidade que nele vive ${ }^{2}$. Entre as décadas de 70 e 80 , esses profissionais de saúde, deram início às atividades no sistema público de saúde, ocorrendo a sua inclusão em creches, escolas, berçários, postos, centros e unidades de saúde, bem como na coletividade ${ }^{3,4}$.

Apesar de a Fonoaudiologia ser uma ciência recente, seu campo de atuação cresce cada dia. Com isso, vem expandindo seu domínio de atuação, havendo um aumento considerável da inserção de profissionais de Fonoaudiologia na Saúde Pública, buscando reorganizar seus serviços que antes eram direcionados apenas à prática clínica individual em ambulatórios, consultórios e clínicas, e desenvolver novas formas de atuação, de modo a contemplar as necessidades dos serviços públicos, em especial no campo da prevenção e promoção 
da saúde, pública e coletiva ${ }^{5-7}$. Neste contexto, o fonoaudiólogo, junta-se a outros profissionais de saúde, levando a perspectiva multidisciplinar e/ou interdisciplinar, proporcionando a atenção integral dos usuários, outras formas de atuação e possibilidades de agir no âmbito da prevenção, promoção, proteção e reabilitação em saúde ${ }^{8,9}$. Questionandose também a prática no âmbito da Saúde Pública em que não só os indivíduos que detêm alto poder aquisitivo apresentam problemas fonoaudiológicos, mas também, os que pertencem às camadas sociais menos favorecidas ${ }^{9}$.

Por meio de pesquisas realizadas nessa área, os fonoaudiólogos vêm definindo o seu campo de atuação frente à promoção de saúde da população de forma consciente, responsável e atuante. Com o objetivo de elaborar e efetivar ações que visem adotar com o objetivo de elaborar e efetivar ações que visem adotar medidas de promoção, prevenção e recuperação da saúde fonoaudiológica da população ${ }^{4,10,11}$. Porém, para que tais profissionais estejam aptos para atuar no serviço público devem ter conhecimentos específicos sobre temas que relacionam fonoaudiologia e saúde coletiva, sendo capaz de identificar alterações fonoaudiológicas de maior importância para a população assistida, elaborar e efetivar ações, buscando soluções cabíveis a realidade e alterações encontradas e por fim adotar as medidas necessárias de prevenção, a fim de proporcionar um atendimento de qualidade 12. Assim, é necessário ter habilidade na percepção das alterações em todas as áreas da Fonoaudiologia, que acometem a população. A Fonoaudiologia a partir da contribuição de todas suas áreas de atuação deve garantir melhorias nas condições de saúde geral e contribuir para a promoção da saúde integral dos pacientes atendidos ${ }^{5,8,13}$. A formação desse profissional deve incluir além da educação multidisciplinar, métodos e técnicas inovadoras e de baixo custo para a realidade da saúde pública a que está inserido. A aprendizagem deve ser um processo contínuo, destacando-se a importância da atualização profissional ${ }^{11,14}$.

A demanda em um serviço público de saúde é numerosa, com isso, o tratamento torna-se complexa a realização de um tratamento de qualidade $e$ com o tempo de duração esperado. Assim, a terapia fonoaudiológica leva um longo período de duração e com poucas previsões de alta ${ }^{15}$.

Pretende-se, contudo, contribuir com esse estudo para um melhor direcionamento quanto ao tipo de assistência prestada, visando uma melhor promoção, proteção, e reabilitação da saúde, e oferecer também, uma contribuição para futuras pesquisas em Fonoaudiologia no âmbito do serviço público.
O objetivo desta pesquisa foi traçar o perfil dos pacientes atendidos pelo setor de Fonoaudiologia de um serviço público do Recife, segundo a faixa etária e gênero dos pacientes, origem dos encaminhamentos e número de queixas por encaminhamentos e queixas fonoaudiológicas mais frequentes.

\section{MÉTODOS}

Este estudo é do tipo transversal, no qual descreve um único momento e observacional (pesquisa documental), pois a pesquisa foi realizada por meio da análise das fichas da Entrevista Inicial do setor de Fonoaudiologia do Instituto de Reabilitação Infantil (IRI), não havendo, portanto contato com os pacientes.

A pesquisa foi realizada no Instituto de Reabilitação Infantil (IRI) na cidade do Recife, sendo uma clínica conveniada com o SUS há 38 anos, atendendo pacientes em todas as faixas etárias e todas as patologias, oferecendo serviços de Fonoaudiologia, Fisioterapia e Psicologia gratuitamente aos usuários do serviço público de saúde. São realizados 100 atendimentos por semana, divididos em 20 pacientes por dia, no setor de Fonoaudiologia.

Participaram desta pesquisa todos os pacientes atendidos no Setor de Fonoaudiologia do Instituto de Reabilitação Infantil, 251 pacientes, tendo seu critério de inclusão os pacientes que apresentaram os dados completos na ficha da entrevista inicial ou que não tivessem de alta fonoaudiológica. Foram excluídos 12 pacientes, pois alguns apresentaram alta fonoaudiológica e outros dados incompletos da ficha da entrevista inicial, esta pesquisa foi realizada no período de Julho de 2007 a maio de 2008.

Para esta coleta de dados, foram utilizados os dados dos prontuários, ou seja, das fichas da entrevista inicial dos pacientes atendidos no setor de Fonoaudiologia do IRI.

As variáveis estudadas consistiram na faixa etária dos pacientes, gênero, queixas fonoaudiológicas apresentadas nas entrevistas iniciais, origem dos encaminhamentos e número de queixas por encaminhamentos, visando traçar o perfil dos pacientes atendidos no setor de Fonoaudiologia do IRI.

A coleta de dados aconteceu no período de 01 de julho a 30 de outubro de 2008, após a aprovação do Comitê de Ética em Pesquisa em seres humanos do Hospital Agamenon Magalhães/Recife - PE, no dia 25/06/2008, conforme a resolução do CONEP 196/96.

A partir da análise das informações contidas nas fichas da entrevista inicial, os dados foram analisados por meio de estatística descritiva com frequência absoluta e relativa, sendo os resultados apresentados por gráficos. 


\section{RESULTADOS}

Foram incluídos nesta pesquisa os dados de $239(100 \%)$ pacientes atendidos no setor de Fonoaudiologia de um serviço público do Recife, evidenciando-se o predomínio, 131 pacientes, na faixa etária de $0-11$ anos (54,81\%) (Figura1) e 146 pacientes do sexo masculino $(61,08 \%)$ (Figura 2).

Em relação à origem dos encaminhamentos, $60(25,01 \%)$ foram realizados por Pediatras e 39 $(16,31 \%)$ por Otorrinolaringologista (ORL), em Outros estão incluídos Ortodontista, Psiquiatra, Buco-maxilo, Assistente social, Pneumologista,
Ortopedista e a própria família, totalizando 28 $(11,71 \%)$ (Figura 3).

A grande maioria dos encaminhamentos, 174 $(72,80 \%)$, apresentou uma única queixa fonoaudiológica (Figura 4), sendo as mais frequentes as alterações de voz, 38 (15,89\%). Nestas estão incluídos: disfonia orgânico-funcional, 24 (10,04\%); disfonia infantil, 11 (4,60\%); puberfonia, um (0,41\%); alteração de ressonância (insuficiência velo-faríngea), um $(0,41 \%)$; e câncer de laringe, um $(0,41 \%)$. Seguidos de desvio fonológico, 34 (14,22\%); atraso de linguagem, 26 (10,87\%); e disfluência, 24 (10,04\%) (Figura 5).

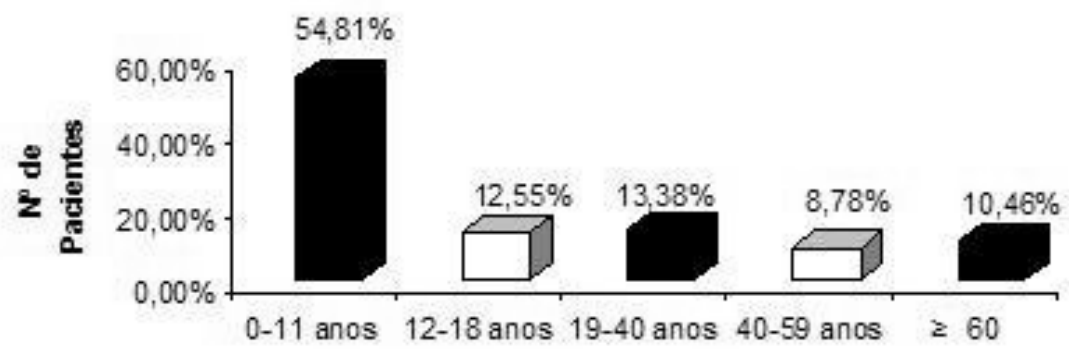

Figura 1 - Número de pacientes de acordo com a faixa etária ( $\mathrm{N}=239)$

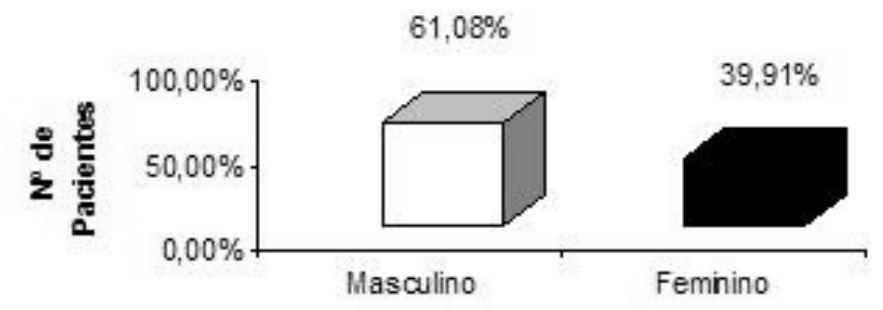

Figura 2 - Número de pacientes de acordo com o gênero ( $\mathrm{N}=239)$

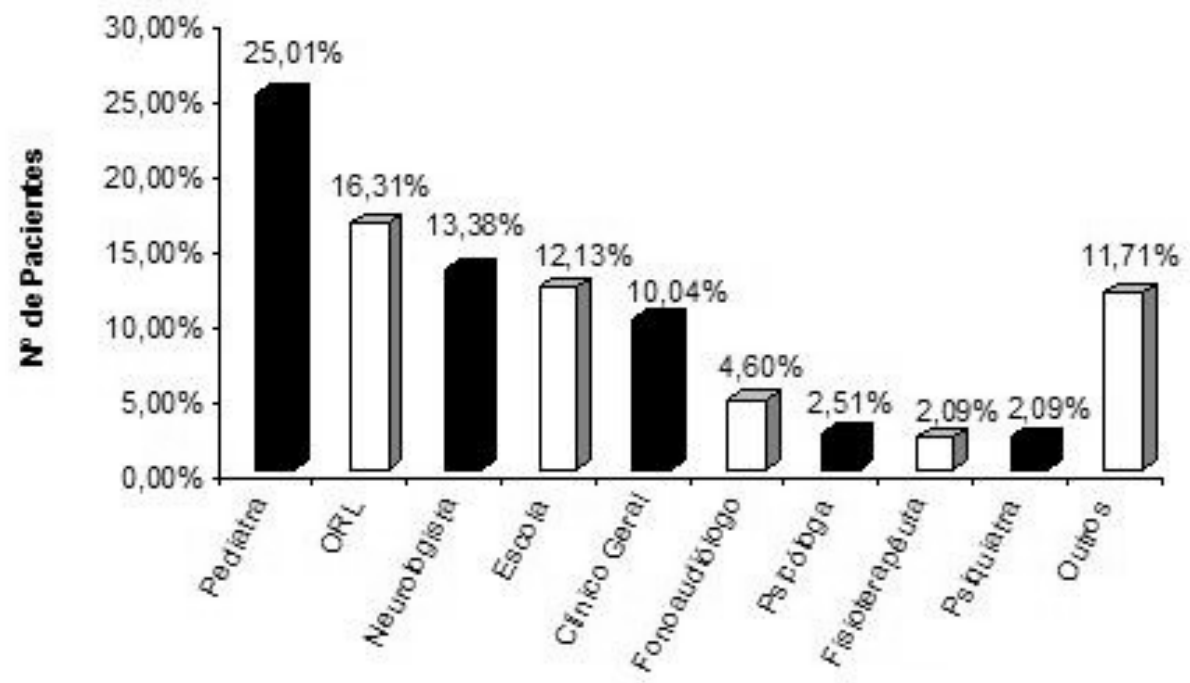

Figura 3 - Origem dos encaminhamentos $(\mathrm{N}=239)$ 


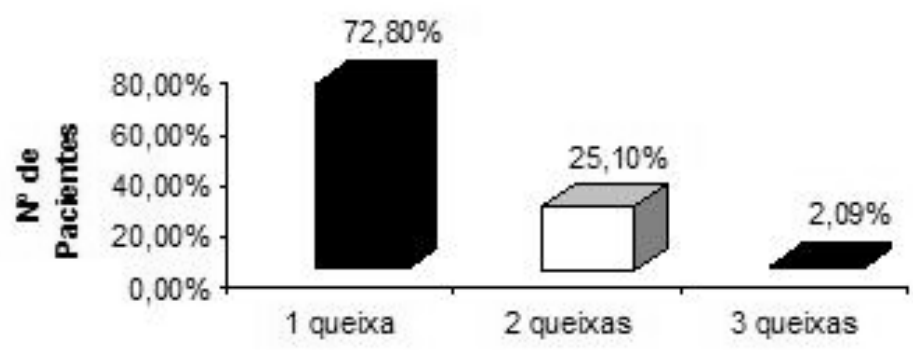

Figura 4 - Número de queixas por encaminhamento (=239)

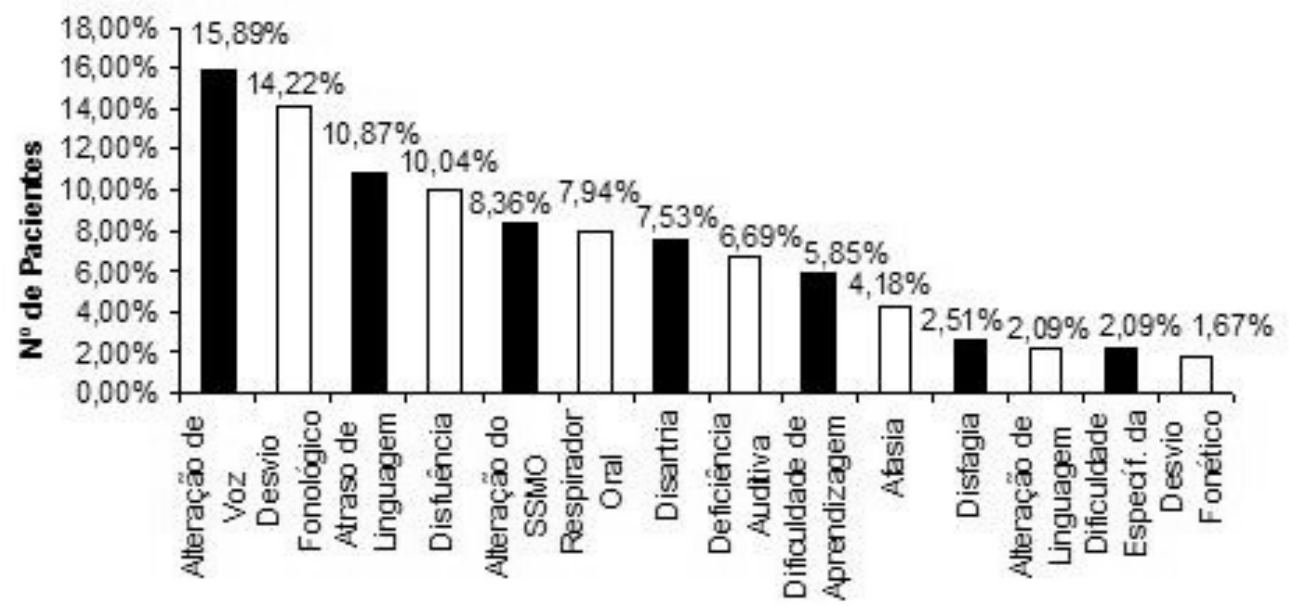

Figura 5 - Queixas fonoaudiológicas mais frequentes $(\mathrm{N}=239)$

\section{DISCUSSÃO}

Observou-se neste estudo que a demanda fonoaudiológica em clínicas conveniadas com o SUS é numerosa, podendo constatar que em menos de um ano foram atendidos 251 pacientes.

Nesta pesquisa a maior demanda fonoaudiológica encontra-se na faixa etária de $0-11$ anos, podendo ser explicado pelo ingresso na fase préescolar e escolar, sendo o período que a criança inicia novas interações sociais e são mais exigidas em nível de ensino/aprendizagem ${ }^{8,16}$.

Além disso, houve predomínio do sexo masculino, vários estudos referem sobre isso, sugerindo esta ocorrência ao fato da aquisição e o desenvolvimento da linguagem entre meninos e meninas parece ocorrer de forma diferente, principalmente devido à forma distinta que ocorre a interação com o meio e a forma de criação ou pelo fato de que o cérebro dos meninos apresenta uma maturação mais lenta do que o das meninas. Entretanto, não há nenhum estudo que comprove a relação entre alterações fonoaudiológicas e a predominância do sexo masculino ${ }^{16-19}$.

Estudos realizados relatam que a escola é um dos maiores responsáveis pelo número de encaminhamentos ${ }^{13,20}$, o que não coincidem com esta pesquisa, em que foi observado que Pediatras e Otorrinolaringologista (ORL) foram os que mais encaminharam.

A presença de uma única queixa fonoaudiológica, ou seja, alteração fonoaudiológica relatada pelos pacientes, foi comumente observada nos encaminhamentos, diferentemente do único estudo encontrado que refere duas queixas fonoaudiológicas associadas ${ }^{13}$.

As alterações de voz e desvio fonológico apresentaram uma maior ocorrência, podendo ser explicado pelo fato dos encaminhamentos serem ocorridos mais por Pediatras e ORL. Estudos relatam que na faixa etária de 5 a 7 anos ocorrem uma maior prevalência de desvio fonológico, podendo ser considerado esperado algumas imprecisões fonêmicas, tornando-se um quadro lingüístico severo após os 6 anos de idade ${ }^{21,22}$.

O profissional de fonoaudiologia pode estar presente em todos os segmentos da Saúde, desde a baixa, como mostra neste estudo, como na média e alta complexidade. O trabalho do fonoaudiólogo realizado junto aos pacientes na atenção básica mostra a importância da sua presença no cotidiano dos postos de saúde ${ }^{23}$. Sendo assim, o 
fonoaudiólogo detém conhecimentos não apenas de problemas relacionados a fala e audição, mas também de saúde e funcionamento do corpo humano como um todo. Incluindo questões culturais, emocionais, físicas, ambientais e econômicas, em outras palavras, pode-se dizer que humanizar sua atuação e perceber que este profissional pode contribuir para a coletividade e não somente para indivíduos ou grupos isolados ${ }^{24}$.

A prevenção, a promoção de saúde e os princípios do SUS são bases norteadoras para a inserção da Fonoaudiologia no contexto da Saúde Pública, podendo estar presente em todos os níveis de atuação ${ }^{10}$. Mas, a visão fonoaudiológica ainda encontrada nos serviços públicos de saúde, principalmente aqueles que não tiveram uma atualização curricular em sua vida acadêmica voltado para promoção de saúde, possui uma visão predominantemente clínica, com a preocupação de atender toda a demanda, que procuram as unidades básicas de saúde, hospitais e centros de referências, entre outros ${ }^{25}$.

Enfim, espera-se que o fonoaudiólogo, como profissional de saúde inserido no serviço público, observe as necessidades e características da população, tendo uma visão crítica em relação às políticas de saúde vigentes.

\section{CONCLUSÃO}

O perfil fonoaudiológico dos pacientes atendidos nesta clínica no Recife - PE, foi caracterizado pela faixa etária de $0-11$ anos, do sexo masculino, com alterações de voz e desvio fonológicos, sendo encaminhados por Pediatras e Otorrinolaringologistas, apresentando em sua maioria única queixa fonoaudiológica.

\begin{abstract}
Purpose: to delineate the profile of patients registered and attended by a Speech Therapy sector in a public service at Recife. Methods: the study was conducted from the collection and analysis of data concerning the origin of referral, age, gender, speech complaints of children, adolescents, adults and elderly, collected from the records of initial interviews. The sample consisted of all individuals registered in the sector of Speech Therapy in a public service at the city of Recife, from July 2007 to May 2008. Results: from 251 patients attending the sector of Speech Therapy in a public service at Recife, 12 were excluded from the search because three were discharged and nine have not had full data. In the tested records, there was a greater number of referral performed by Pediatricians $(25 \%)$, then Otorhinolaryngologist (16\%) and General Clinical (10\%), with the majority of males $(61 \%)$, aged from 0 to 11-year old. According to the complaints, the most frequent was phonological diversion (14\%), followed by language delay (11\%), disfluency $(10 \%)$ and oral respirator $(8 \%)$. Regarding the number of complaints, $72 \%$ had one complaint, $25 \%$ had two complaints and $2 \%$ had three associated complaints. Conclusions: this research showed that the profile seen in the sector of spech therapy of a public service in the city of Recife, although varied, is mostly male, guided by pediatricians, with change of voice, age from 0 to 11 -year old, often featuring a single speech therapy complaint.
\end{abstract}

KEYWORDS: Primary Health Care; Public Health; Public Sector; Health Services; Rehabilitation

\section{REFERÊNCIAS}

1. Natalini G. Princípios Básicos do SUS. In: Associação Paulista de Medicina. SUS: o que você precisa saber sobre o Sistema Único de Saúde. São Paulo: Raiz; 2001. p.21-24.

2. Bernardi APA. Fonoaudiologia na saúde coletiva: uma área em crescimento. Editorial II. Rev. CEFAC. 2007 Abr-Jun; 9(2):146-7.

3. Aquino $F$, Nunes G. Aleitamento materno benefícios fonoaudiológicos à comunidade. [homepage na internet] Guia de fonoaudiólogos no Brasil e artigos de fonoaudiologia; 2007. [acesso em 01 jun 2008]. Disponível em: URL:

$<$ http://www.fonoaudiologos.net/fonoaudiologiasaude-publica-artigos/27-aleitamento-maternobeneficios-fonoaudiologicos-comunidade.html> 4. Dahan D. Atuação fonoaudiológica em Saúde Pública: uma abordagem preventiva. [homepage na internet] Fonoaudiologia.com; 2001 [acesso em 31 maio 2008] Disponível em: URL: <http://www. fonoaudiologia. com/ trabalhos/artigos/artigo-026. $\mathrm{htm}>$ 
5. Souza RPF, Cunha DA, Silva HJ. Fonoaudiologia: a inserção da área de linguagem no sistema único de saúde (SUS). Rev. CEFAC. 2005; 7(4):426-32. 6. Penteado RZ, Chun RYS, Silva RC. Do higienismo às ações promotoras de saúde: a trajetória em saúde vocal. Dist Comun. 2005; 17(1):9-17.

7. Lenz AJ, Gernhardt A, Goulart BNG, Zimmer F, Rocha JG, Vilanova JR, et al. Acolhimento, humanização, e fonoaudiologia: relato de experiência em unidade básica de saúde de Novo Hamburgo (RS). Boletim de Saúde. 2006; 20(2):59-69.

8. Leonelli BS, Fedosse E, Silva RC, Chun RYS, Marin CR. Fonoaudiologia comunitária da UNIMEP: ações fonoaudiológicas em serviços de saúde/ educação. Saúde em Revista. 2003; 5(11):57-63.

9. Campelo LD, Barros PMF, Lima CN, Brito KM, Viana LGO, Veloso MML, et al. Fonoaudiologia na Saúde Pública: uma intervenção para a prevenção da surdez. [homepage na internet] Guia de fonoaudiólogos no Brasil e artigos de fonoaudiologia; 2007. [acesso em 05 maio 2008] Disponível em: URL:

$<$ http://www.fonoaudiologos.net/fonoaudiologiasaude-publica-artigos/25-fonoaudiologia-na-saudepublica-uma-intervencao-para-a-prevencao-dasurdez.html >

10. Penteado RZ, Servilha EAM. Fonoaudiologia em saúde pública/coletiva: compreendendo prevenção e o paradigma da promoção da saúde. Dist Comun. 2004; 16(1):107-16.

11. Pereira MTJG. Fonoaudiologia: uma vivência em saúde pública. [monografia]. São Paulo (SP): CEFAC - Pós-Graduação em Saúde e Educação; 1999.

12. Befi-Lopes DM. A inserção da fonoaudiologia na atenção primária à saúde. In: Befi-Lopes DM. Fonoaudiologia na atenção primária à saúde. 1. ed. São Paulo: Lovise; 1997. p.15-35.

13. César AM, Maksud SS. Caracterização da demanda de fonoaudiologia no serviço público municipal de Ribeirão das Neves - MG. Rev. CEFAC. 2007; 9(1):133-8. dx.doi.org/10.1590/ S1516-18462007000100017

DOI: 10.1590/S1516-18462009005000063

RECEBIDO EM: 16/12/2008

ACEITO EM: 15/09/2009

Endereço para correspondência:

Percy Maria de Lima Barros

Av. Conselheiro Aguiar, 3743 ap. 14

Recife - PE

CEP: 51021-020

E-mail: percylimab @ hotmail.com
14. Goulart BNG, Chiari BM. Testes de rastreamento $x$ testes de diagnóstico: atualidades no contexto da atuação fonoaudiológica. Pró-Fono. 2007; 19(2):223-32.

15. Castro EM. A fonoaudiologia e o cotidiano na saúde pública. [homepage na internet]. Fonoaudiologia.com; 2001. [acesso em 31 maio de 2008] Disponível em: URL:

<http://www.fonoaudiologia.com/trabalhos/artigos/ artigo-034.htm>

16. Gonçalves CGO, Lacerda CBF, Perotino S, Mugnaine AMM. Demanda pelos serviços de fonoaudiologia no município de Piracicaba: estudo comparativo entra a clínica escola e o atendimento na prefeitura municipal. Pró-Fono. 2000; 12:61-6.

17. Freire RM. Fonoaudiologia em saúde pública. Rev Saúde Pública. 1992; 26(3):179-84.

18. Morales MV, Mota HB, Keske-Soares M. Consciência fonológica: desempenho de crianças com e sem desvios fonológicos evolutivos. PróFono. 2002; 14(2):153-64.

19. Goulart BNG, Chiari BM. Prevalência de desordens de fala em escolares e fatores associados. Rev Saúde Pública. 2007; 41(5):726-31.

20. Hage SRV, Faiad LNV. Perfil de pacientes com alteração de linguagem atendidos na clínica de diagnóstico dos distúrbios da comunicação. Rev. CEFAC. 2005; 7(4):433-9.

21. Wertzner HF. Articulação e suas alterações. In: Kudo AM, Marcondes E, Lins MLF, Moriyama LT, Guimarães MLLG, Juliani RCTP, et al. Fisioterapia, fonoaudiologia e terapia ocupacional em pediatria. 2. ed. São Paulo: Sarvier; 1994. p. 108-14.

22. Andrade CRF. Prevalência das desordens idiopáticas da fala e da linguagem em crianças de um a onze anos de idade. Rev Saúde Pública. 1997; 31(5):495-501.

23. Formiga R. Nasf: fonoaudiologia conquista espaço na saúde publica. J Cons Fed Fonoaudiol. 2008; 9(36):4-5.

24. Lipay MS, Almeida EC. A fonoaudiologia e sua inserção na saúde pública. Rev Ciênc Med. 2007; 16(1):31-41.

25. Cruz MS, Oliveira LR, Carandina L. Inquéritos de saúde e fonoaudiologia. Rev. CEFAC. 2009; 11(1):166-72. dx.doi.org/10.1590/ S1516-18462009005000009 